\title{
Face Recognition Based on Polar Frequency Features
}

\author{
YOSSI ZANA and ROBERTO M. CESAR, JR \\ University of São Paulo
}

\begin{abstract}
A novel biologically motivated face-recognition algorithm based on polar frequency is presented. Polar frequency descriptors are extracted from face images by Fourier-Bessel transform (FBT). Next, the Euclidean distance between all images is computed and each image is now represented by its dissimilarity to the other images. A pseudo-Fisher linear discriminant was built on this dissimilarity space. The performance of discrete Fourier transform (DFT) descriptors and a combination of both feature types was also evaluated. The algorithms were tested on a 40- and 1196-subjects face database (ORL and FERET, respectively). With five images per subject in the training and test datasets, error rate on the ORL database was 3.8, 1.25, and 0.2\% for the FBT, DFT, and the combined classifier, respectively, as compared to $2.6 \%$ achieved by the best previous algorithm. The most informative polar frequency features were concentrated at low-to-medium angular frequencies coupled to low radial frequencies. On the FERET database, where an affine normalization preprocessing was applied, the FBT algorithm outperformed only the PCA in a rank recognition test. However, it achieved performance comparable to state-of-the-art methods when evaluated by verification tests. These results indicate the high informative value of the polar frequency content of face images in relation to recognition and verification tasks and that the Cartesian frequency content can complement information about the subjects' identity, but possibly only when the images are not prenormalized. Possible implications for human face recognition are discussed.
\end{abstract}

Categories and Subject Descriptors: I.4 [Image Processing and Computer Vision]: Feature Measurement; Image Representation, Object Recognition; I5 [Pattern Recognition]: Feature Evaluation and Selection, Pattern Analysis, Computer Vision

General Terms: Face Recognition, Human Perception, Algorithms

Additional Key Words and Phrases: Fourier-Bessel transform, discrete Fourier transform

\section{INTRODUCTION}

Face recognition is a highly complex task due to the many possible variations of the same subject in different conditions, like luminance and facial expressions, and the three-dimensional nature of the head. Many developers of face-recognition algorithms adopted a biologically inspired approach in solving this problem [for a review, see Calder et al. 2001], thus contributing both to understand human face-processing and to build efficient face-recognition technologies. Recent developments in the neurophysiology field inspired the development of a high-performance face-recognition approach described in the present paper. The proposed approach is based on features that may be analogous to those extracted by the human visual system (HVS) from the visual scene. In particular, we evaluated the performance of a face-recognition algorithm whose primary features were the magnitude of Cartesian or radial and angular components of images of faces. To the best of our knowledge, we show that the proposed approach is comparable to the state-of-the-art algorithms tested on the same databases. Possible implications for human face recognition are also discussed.

Authors' addresses: Department of Computer Science - IME, University of São Paulo, São Paulo - SP 05508-900, Brazil; email: [zana,roberto-cesar]@ime.usp.br.

Permission to make digital or hard copies of part or all of this work for personal or classroom use is granted without fee provided that copies are not made or distributed for profit or direct commercial advantage and that copies show this notice on the first page or initial screen of a display along with the full citation. Copyrights for components of this work owned by others than ACM must be honored. Abstracting with credit is permitted. To copy otherwise, to republish, to post on servers, to redistribute to lists, or to use any component of this work in other works requires prior specific permission and/or a fee. Permissions may be requested from Publications Dept., ACM, Inc., 1515 Broadway, New York, NY 10036 USA, fax: +1 (212) 869-0481, or permissions@acm.org. (c) 2006 ACM 1544-3558/06/0100-0062 $\$ 5.00$

ACM Transactions on Applied Perception, Vol. 3, No. 1, January 2006, Pages 62-82. 


\section{BACKGROUND AND PREVIOUS WORKS}

\subsection{Spatial Analysis in Polar Coordinates}

Most of the current face-recognition algorithms are based on feature extraction from a Cartesian perspective, typical to most analog and digital imaging systems. The primate visual system, on the other hand, is known to process visual stimuli logarithmically. For example, biological evidences indicate that the retinal image is retinothopically mapped onto area V1 of the visual cortex in a log-polar manner, i.e., image representation in the cortex is negatively correlated with retinal cell eccentricity [Schwartz 1977]. This property led to a formulation of a spatial log-polar transformation [Schwartz 1980] in which a Cartesian image was resampled as a logarithmic function of the distance from the center. This transformation was explored by several feature-detection [Grove and Fisher 1996; Lim et al. 1997; Gomes and Fisher 2003] and face-detection [Hotta et al. 1998; Jurie 1999; Chien and Choi, 2000] investigators. The log-polar transformation was also used in face-recognition systems [Tistarelli and Grosso, 1998; Minut et al. 2000; Escobar and Ruiz-de-Solar 2002; Smeraldi and Bigun 2002]. One of the disadvantages of this feature-extraction method is the rough representation of peripheral regions. The HVS compensates this effect by eye saccades, moving the fovea from one point to the other in the scene. Similar approach was adopted by the face-recognition systems of Tistarelli and Gross [1998] and Smeraldi and Bigun [2002]. Escobar and Ruiz-del-Solar [2002] applied the log-polar transformation at a single location, but the subsequent step (Gabor jets filtering) required manual localization of 16 fiducial points in the face image.

Although the previous algorithms that use log-polar transformation well simulate the retinal sampling resolution, they are usually followed by local analysis and do not provide any information about global patterns. An alternative representation of an image in the polar frequency domain is the twodimensional Fourier-Bessel transform [Bowman 1958; Rosental et al. 1982]. This transform found several applications in analyzing patterns in a circular domain [Zwick and Zeitler 1973; Guan et al. 2001; Fox et al. 2003], but was seldom exploited for image recognition. One of such rare examples is the work of Cabrera et al. [1992], who applied the FBT to create descriptors of contour segments from images.

The paper is organized as follows: in the next section, we briefly introduce the reader to the primary spatial processing by the HVS and to the main face-recognition algorithms tested on the face databases used in our experiments. In Section 3 the discrete Fourier transform (DFT) and the Fourier-Bessel Transform (FBT) for face-image analysis is described. The proposed face-recognition algorithms are introduced in Section 4. We present the experimental results on two widely used face databases in Section 5. In the last section, we discuss the results and ongoing work for possible future improvements.

\subsection{Human Spatial Processing}

In the early stages of the human visual processing, specialized neurons act as filters for the visual image. Such neurons are tuned to specific spatial frequencies and locations in the visual field. The activity of cortical simple cells is well described by linear models [De Valois and De Valois 1990; Itti et al. 2000]. However, the local information provided in the earlier cortical stages must be further processed to extract global shape information. For instance, neurons in the inferotemporal cortex tuned to complex shapes, such as faces, have been reported [Perret et al. 1982]. Moreover, the response of these facespecific neurons was correlated with two-dimensional patterns, but not with three-dimensional shapes or any internal configural relations of the pattern [Young and Yamane, 1992].

Since the pioneer work of Kelly [1960], who suggested probing the HVS with symmetrically circular stimuli, many investigators of global shape processing in the early visual stages used circular shapes and found evidence of polar visual form processing. Electrophysiological experiments showed that cells in the LGN, V1, V2, and V4 cerebral areas in monkeys are specifically sensitive to Cartesian, polar, and 

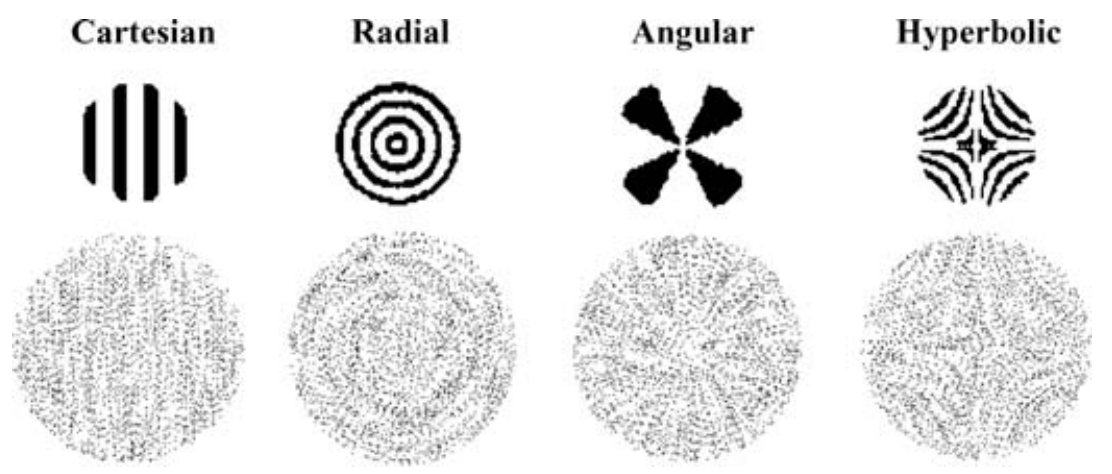

Fig. 1. Examples of stimuli defined in four different coordinate systems. Upper row: Stimuli used to study the response specificity of cells in the V4 cerebral area of monkeys [Gallant et al. 1996]. Lower row: Glass patterns used for measuring of the detectability of dot patterns [Wilson and Wilkinson 1998].

hyperbolic stimuli [Gallant et al. 1993, 1996; Mahon and De Valois 2001]. Moreover, psychophysical measurements of Glass dot patterns detection thresholds as a function of the stimulated area showed global pooling of orientation information in the detection of angular and radial dot patterns [Wilson and Wilkinson 1998]. Thus, it is evident that information regarding the global polar content of images is effectively extracted by and available to the HVS. Examples of the stimuli used by these investigators are presented in Figure 1.

Does polar form analysis help in face-recognition tasks? To answer this question we need to evaluate the amount of information about the subject identity carried by polar components. Furthermore, we have to verify that the information is actually used by the HVS to recognize faces. In the current paper, we describe an attempt to answer the former question by developing a new efficient face-recognition method.

\subsection{Previous Face-Recognition Algorithms}

One of the fastest and most used face-recognitions algorithms is the eigenfaces scheme [Turk and Pentland, 1991]. The first eigenvectors (principal components) obtained from a set of training images form the basic representation in that approach. Eigenfaces, the projection of the images onto these eigenvectors, are used as classification features. At the testing stage, unlabeled (probe) images are projected onto the eigenvector basis and compared to the learned images in the new face space. Principal Component Analysis (PCA) was used to model facial expression recognition [Calder et al. 2001] and several known face effects, like distinctiveness, caricature, and "other-race" [see Calder et al. 2001, for a detailed review]. There is also an interesting interpretation of the eigenfaces algorithm related to neurons specifically tuned to face images and to biological autoassociative memory models [Abdi 1988; O'Toole et al. 1995]. The classic eigenfaces method is a good example of a global (holistic) algorithm, in contrast to feature-based (local) algorithms, where the image is encoded as a whole. A major drawback of the method is its requirement of a precise spatial normalization of the face parts, such that the eyes, mouth, etc., are scaled and registered at the same spatial coordinates [Craw and Cameron 1991].

Also taking a holistic approach, Hafed and Levine [2001] explored the discrete cosine transform (DCT) as a means of feature extraction for face classification. Analogous to the Fourier transform, the DCT maps an image from the spatial to the frequency domain, extracting the frequency components. However, it differs from the DFT in using only real coefficients. The biological appeal of the DCT features lies in the spatial to (Cartesian) frequency domain transformation that is believed to occur in the HVS [Campbell and Robson 1968]. 
An algorithm developed in the University of Southern California proposed the Gabor wavelets image transform as mean of feature extraction [Lades et al. 1993; Wiskott et al. 1997]. This transform can be seen as analogous to the multiscale multiorientation spatial analysis observed in the V1 cortical area [Hubel and Wiesel 1968; Movshon et al. 1978; De Valois et al. 1982]. In contrast to the global analysis of the DCT, the Gabor wavelets features are extracted at fiducial points, corresponding to anatomically identifiable nodes in a geometric model (graph) of the face. Probe images are projected onto the Gabor jets and compared, through elastic graph matching, to the learned images based on both the extracted coefficients at the corresponding locations and distances between the formed graphs. This method requires manual definition of the grid structure, but a fully automatic version was published recently [Arca et al. 2003].

Etemad and Chellappa [1997] used a hybrid approach in which images were initially represented by facial features in the spatial domain as well as by the wavelet transform. In a second stage, new features were extracted through linear discriminant analysis (LDA). In contrast to PCA, the features are projected in LDA such that the (between-subjects variation)/(within-subjects variation) ratio is maximized. Moghaddam et al. [2000] proposed a Bayesian generalization of the LDA method where, instead of matching images through a Euclidean-based similarity measurement, the a posteriori probability of the difference between images is estimated considering the within- and between-subject variation in the training set. All computations were done in a PCA dimensionality reduced space.

Lawrence et al. [1997] proposed a different hybrid algorithm that combined local image sampling, a self-organizing map (SOM) neural network, and a convolutional neural network (CN). The SOM provides a quantization of the image samples into a topological space, where inputs that are nearby in the original space preserve proximity in the output space, while the $\mathrm{CN}$ network extracts successively larger features in a hierarchical set of layers. The SOM topological mapping can be associated to the retinotopic maps found in the visual cortex [Obermayer et al. 1991], while the hierarchical layers of the CN networks are analogous to the multiscale spatial sampling of the HVS [De Valois and De Valois 1990].

As we expect our algorithm to be comparable not only from a biological perspective, but also from the practical point-of-view, nonbiologically motivated algorithms are also cited here. One of these algorithms was designed by Samaria and Harter [1994], being based on hidden Markov models (HMMs) of a spatial top-down sampling. Samaria [1994] also published an extended version with pseudo two-dimensional HMMs. Another algorithm of interest is the ARENA [Sim et al. 2000], based on dimensionality reduction by simply averaging nonoverlapping regions in the images. It then matches training and probe images basically by counting the number of components that differ in value (the $L_{p}^{*}$ dissimilarity measurement).

\section{IMAGE TRANSFORMS}

\subsection{Discrete Fourier Transform}

Spatial frequency analysis in Cartesian coordinates is traditionally done by applying the twodimensional (2D) DFT and was already used for face recognition. For example, Akamatsu et al. [1991] applied the eigenfaces method to the magnitude of the Fourier spectrum as a mean of reducing variability because of changes in head orientation and shifting. The DFT is a well-known analysis tool and will be briefly described. The equation to compute the DFT on an $M \times N$ size image is

$$
F(u, v)=\frac{1}{\sqrt{M N}} \sum_{x=0}^{M-1} \sum_{y=0}^{N-1} f(x, y) e^{-j 2 \pi\left(\frac{u x}{M}+\frac{v y}{N}\right)}
$$

where $u$ and $v$ are the coordinates in the Fourier domain and $x$ and $y$ the coordinates in the space domain. The DFT deals with complex numbers that represent the magnitude and phase of the sine and 


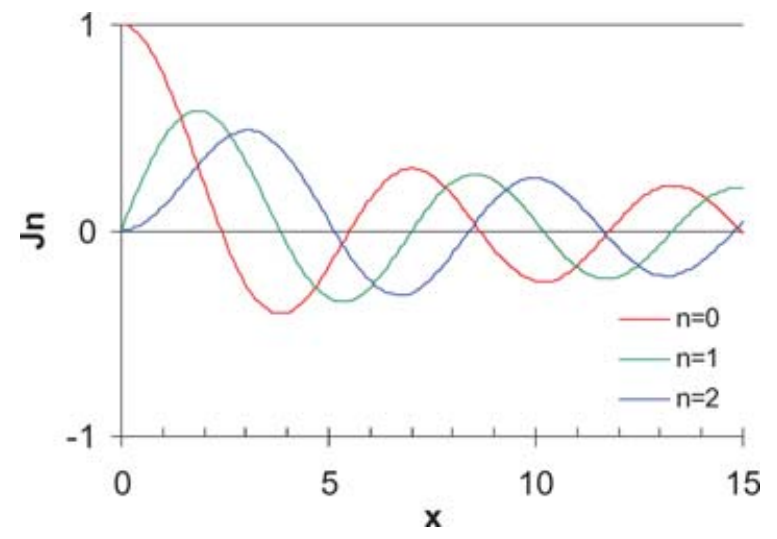

Fig. 2. A plot of the Bessel functions of the first kind of order 0,1 , and 2.

cosine waves in the Fourier formula. However, in the present study, only the magnitude $|F(u, v)|$ was considered.

\subsection{Fourier-Bessel Transform}

Let $f(x, y)$ be the face image. The FBT analysis starts by converting the image pixels description from Cartesian $(x, y)$ to polar $(r, \theta)$ coordinates. Let $\left(x_{0}, y_{0}\right)$ be the origin of the Cartesian image. The polar coordinates necessary to obtain the new image representation $f(r, \theta)$ are defined as

$$
\theta=\tan ^{-1}\left(\frac{y-y_{0}}{x-x_{0}}\right)
$$

and

$$
r=\sqrt{\left(x-x_{0}\right)^{2}+\left(y-y_{0}\right)^{2}}
$$

For square images, the considered maximum radius was the distance from the center of the image to one of the corners. Points outside the original image were discarded. Radial resolution was fixed at one pixel width, but the angular resolution could be varied by increasing or reducing of the number of sampled radii. The intensity of each point of the $f(r, \theta)$ function was determined by bilinear interpolation, combining the values of the four closest pixels weighted by their relative proximity to the reference point [Pratt 1991]. Although this is a linear-polar transformation, in contrast to the aforementioned log-polar transformation, the central area of the Cartesian image is more densely sampled than the periphery. in practice, especially at the high angular resolution used in the current study, because of the limited resolution and discrete nature of digital images,.

The FBT notation and definitions follow Bowman [1958] and Spanier and Oldham [1987]. The $f(r, \theta)$ function is than represented by the Fourier-Bessel series. The Bessel function of the first kind of order $n$ is defined by

$$
J_{n(x)}=\left(\frac{x}{2}\right)^{n} \sum_{k=0}^{\infty} \frac{\left(\frac{-x^{2}}{4}\right)^{k}}{k ! \Gamma(n+k+1)}
$$

where $\Gamma(x)$ is the gamma function. Figure 2 shows a graphical representation of three Bessel functions. 

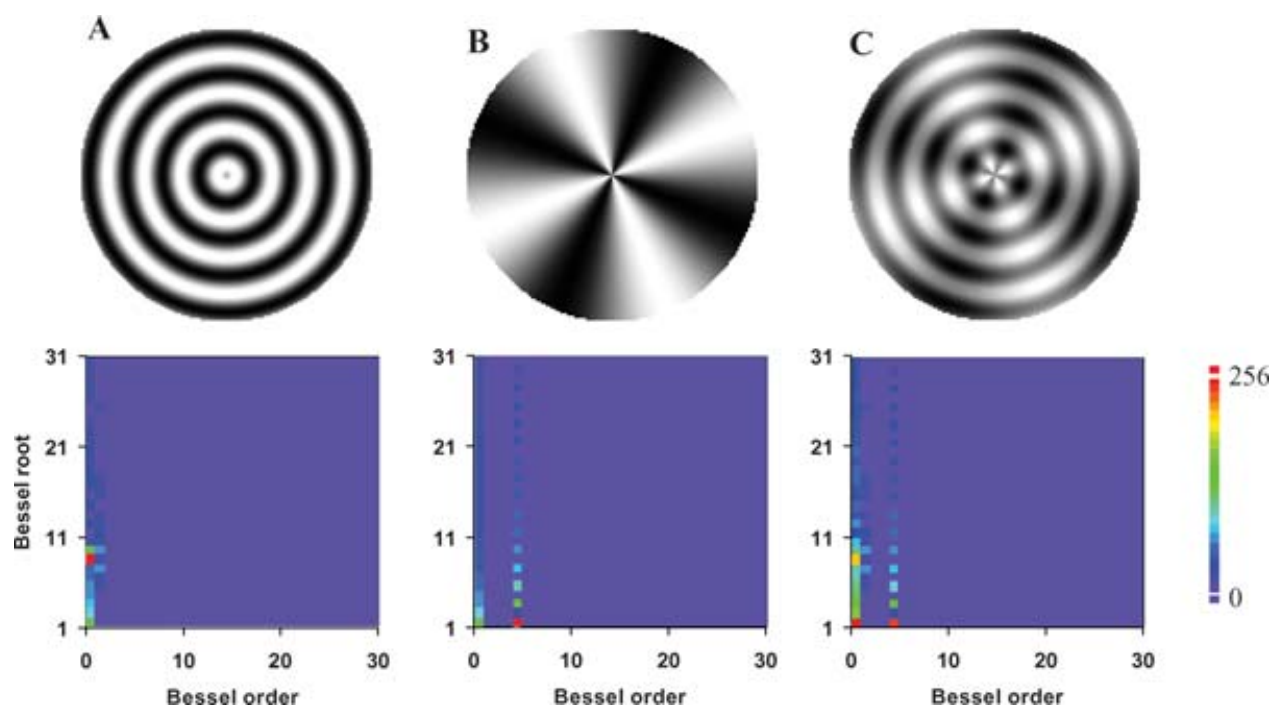

Fig. 3. Examples of FBT of (A) an eight radial cycles image, (B) a four angular cycles, (C) and an image of the average of these images. Image size was $131 \times 131$ pixels. Angular resolution was $0.5^{\circ}$. The modulus of the FBT coefficients is presented in colored levels (red indicates the highest value).

The two-dimensional Fourier-Bessel series is defined as

$$
f(r, \theta)=\sum_{i=1}^{\infty} \sum_{n=1}^{\infty} A_{n, i} J_{n}\left(\alpha_{n, i} r\right) \cos (n \theta)+\sum_{i=1}^{\infty} \sum_{n=1}^{\infty} B_{n, j} J_{n}\left(\alpha_{n, i} r\right) \sin (n \theta)
$$

where $f(R, \theta)=0$ and $0 \leq r \leq R . \alpha_{i}$ is the $i$ th root of the $J_{n}$ function, i.e., the zero crossing value satisfying $J_{n}\left(\alpha_{n}, i\right)=0 . R$ is the radial distance to the edge of the image. The orthogonal coefficients $A_{n, i}$ and $B_{n, j}$ are given by

$$
\begin{gathered}
A_{0, i}=\frac{1}{\pi R^{2} J^{2}{ }_{1}\left(\alpha_{n, i}\right)} \int_{\theta=0}^{\theta=2 x} \int_{r=0}^{r=R} f(r, \theta) r J_{n}\left(\frac{\alpha_{n, i}}{R} r\right) d r d \theta \quad \text { if } B_{0, i}=0 \text { and } n=0 \\
{\left[\begin{array}{l}
A n, i \\
B n, i
\end{array}\right]=\frac{2}{\pi R^{2} J^{2}{ }_{n+1}\left(\alpha_{n, i}\right)} \int_{\theta=0}^{\theta=2 \pi} \int_{r=0}^{r=R} f(r, \theta) r J_{n}\left(\frac{\alpha_{n, i}}{R} r\right)\left[\begin{array}{l}
\cos (n \theta) \\
\sin (n \theta)
\end{array}\right] d r d \theta \quad \text { if } n>0}
\end{gathered}
$$

Some FBT examples are presented in Figure 3. In the original images, a sine function is plotted in radial (eight cycles per image) or angular (four cycles per $360^{\circ}$ ) coordinates, and the third (right) image is the average of both images. Firstly, we transformed the coordinates of the pixels from Cartesian to polar using Eq. (1) and (2), with a $0.5^{\circ}$ angular resolution (i.e., 720 radii). We calculated the coefficients $\mathrm{A}$ and B using Eqs. (5) and (6), limiting $n$ to 30 Bessel order and $i$ to 30 Bessel root. These coefficients represent the magnitude of a spatial variation in polar coordinates [Fox 2000; Guan et al. 2001]. For example, the 0th order, 8th root, and 4th order, 1st root coefficients represent the magnitude of a pure eight radial cycles or four angular cycles pattern, respectively. Similarly, the 4th order, 8th root coefficient represents a pattern, which is the product of these two forms. The A and B coefficients represent the same patterns, but in opposite phase. We plotted the modulus of A and B in Figure 3 for the sake of better visualization. In this spectrum plot, the Bessel order columns represent the relative magnitude 


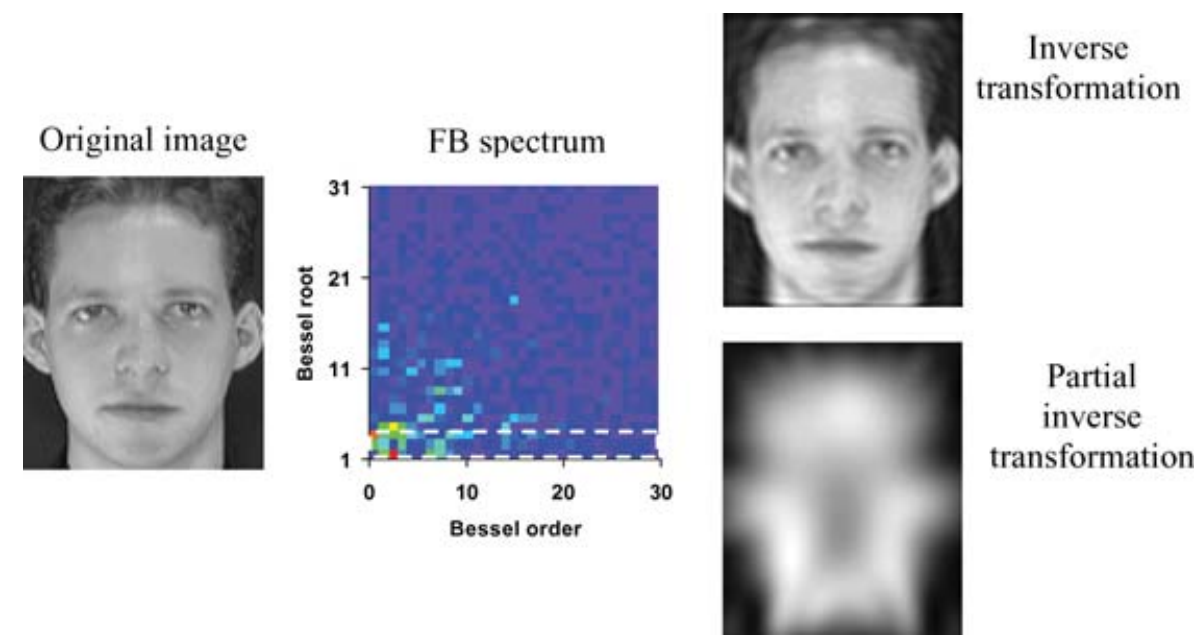

Fig. 4. FBT of a face image from the ORL database. Inverse transforms were derived from the full FB spectrum (upper image) or only up to the 3 rd Bessel root (lower image). The dashed line limits the FB coefficients used for the partial inverse transformation. The lowest coefficient in the spectrum image was set to minimum in order to improve the visibility of the other coefficients.

of the angular frequency, while the Bessel root rows represent the radial frequency. It can be noted that the FBT of the images correctly indicates the principal radial and angular components. The inverse transform images can be calculated from the coefficient matrixes (not shown in the figure).

\section{ALGORITHMS}

\subsection{Feature Extraction}

Dataset handling, training, and tests were done with the Matlab PRTools toolbox [Duin 2000]. After a preprocessing stage (detailed below), images were transformed by a FBT up to the 30th Bessel order and 3rd root with angular resolution of $0.5^{\circ}$, thus leading to 186 coefficients. DFT analysis extracted a total of 1200 magnitude coefficients, corresponding to up to 19.5 cycles per image. The number of DFT coefficients was determined by the optimal performance in preliminary tests on the ORL database.

Figure 4 shows an example of the FBT spectrum (extended to the 30th Bessel root) of a face image from the ORL database [Samaria and Harter 1994]. The spectrum is relatively complex, but with predominance of low-frequency components. From the blurred aspect of the face inverse transform using all the coefficients, it can be noted that the FBT represents low to middle range, but not high, frequencies well. This is a consequence of using a limited number of Bessel orders and roots. Increasing the number of coefficients would widen the sampled frequency range, but also the computation time. However, although the original image cannot be exactly reconstructed (Figure 4), only a limited frequency range is necessary to achieve good recognition rates, as shown below. In the rest of this section, we will refer to the Fourier or Fourier-Bessel transformed images as just images.

\subsection{Dissimilarity Space}

We built a dissimilarity space $D(t, t)$ defined as the Euclidean distance between all training images, where $t$ is the training image. In this space, each object is represented by its dissimilarity to all objects (Table I). This approach is based on the assumption that the dissimilarities of similar objects to "others" is about the same. Dissimilarity space for pattern recognition was first formulated by Duin et al. [1997] and successfully used with the pseudo-Fisher linear discriminant (PFLD) on the ORL face database 
Table I. An Example of a Dissimilarity Space

\begin{tabular}{l|c|c|c}
\multicolumn{4}{|c}{$D(t, t)^{a}$} \\
\hline & Image 1 & Image 2 & Image 3 \\
\hline Image 1 & 0.0 & 0.8 & 0.3 \\
Image 2 & 0.8 & 0.0 & 0.5 \\
Image 3 & 0.3 & 0.5 & 0.0 \\
\hline
\end{tabular}

${ }^{a}$ The value in each cell indicates the Euclidean distance between the corresponding images. In this space, each image is represented by its dissimilarity to all images, i.e., by the values in the corresponding column.

[Pekalska and Duin 2000]. However, while Pekalska and Duin [2000] represented the $256 \times 256(6,5536)$ pixel intensity values in the dissimilarity space, we used 186 coefficients of the FBT. Among other advantages of this representation space, by fixing the number of features (or dimensions) to the number of objects, it avoids a phenomenon known as "peaking" [Raudys and Pikelis 1980], where recognition performance is degraded as a consequence of small number of training samples, as compared to the number of features.

\subsection{Classifier and Testing}

Test images were classified based on a PFLD using a two-class approach. A Fisher linear discriminant (FLD) is obtained by maximizing the Fisher criterion [Fukunaga 1990], i.e., the between subjects variation/within subjects variation ratio. Here we used a minimum-square error classifier implementation [Scurichina and Duin 1996], which is known to be equivalent to the FLD for two-class problems [Fukunaga, 1990]. In these cases, after shifting the data such that it has zero mean, the FLD can be defined as

$$
g(D(x, t))=\left[D(x, t)-\frac{1}{2}\left(\mathrm{~m}_{1}-\mathrm{m}_{2}\right)\right]^{T} S^{-1}\left(\mathrm{~m}_{1}-\mathrm{m}_{2}\right)
$$

where $D(x, t)$ is a probe image, $\mathrm{S}$ is the pooled covariance matrix, and $m_{i}$ stands for the mean of class $i=1,2$. $D(x, t)$ is classified as corresponding to class-1, if $g[D(x, t)] \geq 0$ and to class-2 otherwise. However, as the number of training objects and dimensions is the same in the dissimilarity space, the sample estimation of the covariance matrix $\mathrm{S}$ becomes singular, and the classifier cannot be built. One solution to the problem is to use a pseudo inverse and augmented vectors [Scurichina and Duin 1996]. Thus, Eq. (1) is replaced by

$$
g(D(x, t))=(D(x, t), 1)(D(t, t), I)^{(-1)}
$$

where $(D(x, t), I)$ is the augmented vector to be classified and $(D(t, t), I)$ is the augmented training set. The inverse $(D(t, t), I)^{(-1)}$ is the Moore-Penrose pseudo inverse which gives the minimum norm solution. The pseudo inverse relies on the singular value decomposition of the matrix $[D(t, t), I]$ and becomes the inverse of $[D(t, t), I]$ in the subspace spanned by the eigenvectors corresponding to the nonzero eigenvalues. The classifier is found in this subspace [Pekalska and Duin 2000].

The current $L$-classes problem can be reduced and solved by the two-classes solution described above [Allwein et al. 2000; Duin 2000]. The training set was splitt into $L$ pairs of subsets, each pair consisting of one subset with images from a single subject and a second subset formed from all the other images. A PFLD was built for each pair of subsets. A probe image was tested on all $L$ discriminant functions and assigned to the subject that gave the largest posterior probability.

\subsection{Combining Classifiers}

The FBT- and DFT-based algorithms classified the test images independently. Although both orthogonal bases can lead to a perceptually good reconstruction, they encode different type of information 


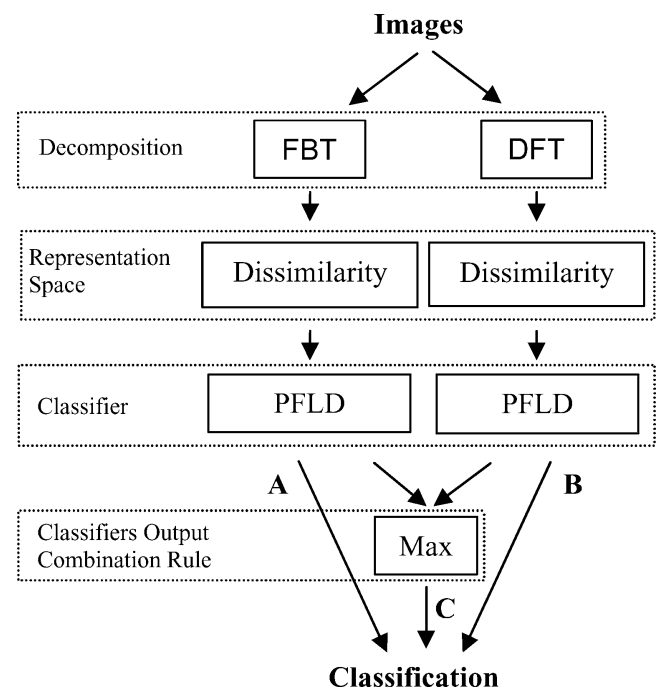

Fig. 5. Block diagram of the three face-recognition algorithms: (A) FBT only, (B) DFT only, and (C) FBT + DFT through maximum rule.

(Cartesian vs. polar frequency content). To evaluate the correlation between the classification errors made by the two algorithms, the soft output of the classifiers were combined by a maximum rule before labeling the images, i.e., the final assignment was determined by the classifier that gave the highest normalized output [Kittler et al. 1998].

Figure 5 present a schematic description of the methods presented in this paper, i.e., (A) FBT only, (B) DFT only, and (C) FBT + DFT through maximum rule.

\section{EXPERIMENTAL RESULTS}

We chose to test our algorithm on two distinct databases: the ORL [Samaria and Harter 1994] and the FERET [Phillips et al. 1998]. The former is a small set with only 40 subjects. However, 10 sample images from each subject, taken with small variations (see details below), are available. The FERET set includes images from thousands of subjects, but only a few samples from each. Both datasets were used as test platforms for many algorithms, making ranking comparisons easier.

\subsection{The ORL Database}

5.1.1 Database and Performance Evaluation. The ORL face database consists of 400 images collected from 40 people. Most of the subjects were 20-35 years old. The face images were $92 \times 112$ pixels with 8-bit gray levels. They included variations in facial expression, luminance, scale and viewing angle, and were shot at different times. Limited side movement and tilt of the head were allowed. Subjects were captured with and without glasses. These characteristics introduce difficulties to correct recognition and make the database particularly interesting. All images were manually cropped and rescaled to the final resolution by the authors of the ORL database [Samaria and Harter 1994]. No further graphical preprocessing was applied here. Figure 6 shows an example of an image set of one subject. Error rate was calculated as the percentage of the misclassified images and each test was repeated 10 times.

5.1.2 General Performance and Learning Rate. Figure 7 shows the final error rates of the three algorithms and a reproduction of results from previously published papers obtained with different models, but where the same database, sampling and testing methodology were used [Samaria and 


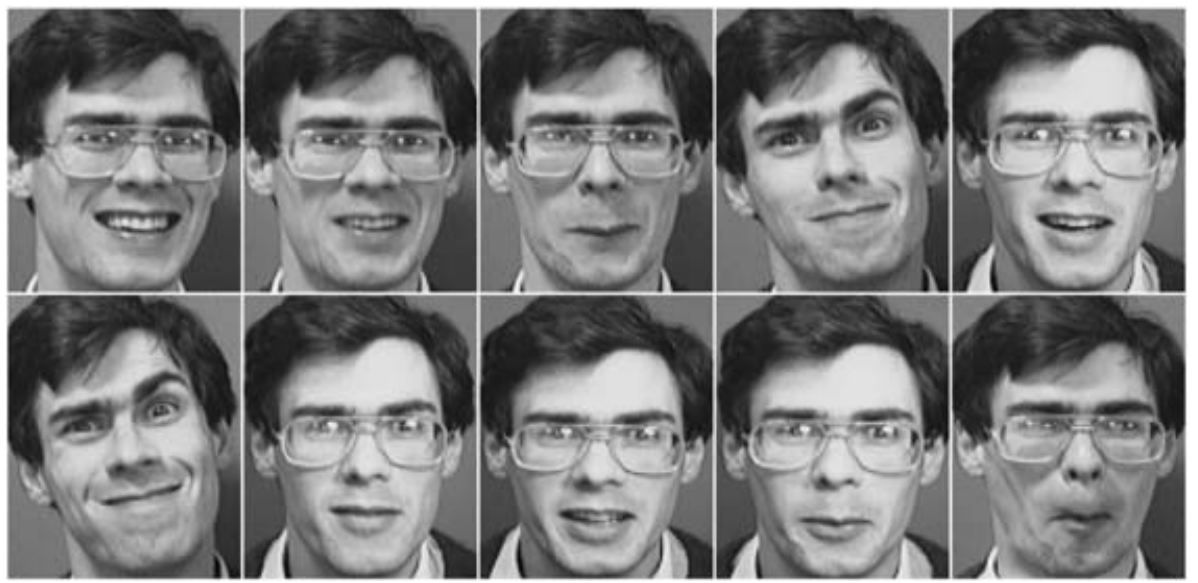

Fig. 6. Images from one subject of the ORL database. There are variations in illumination, head position, facial expressions, and occlusion.

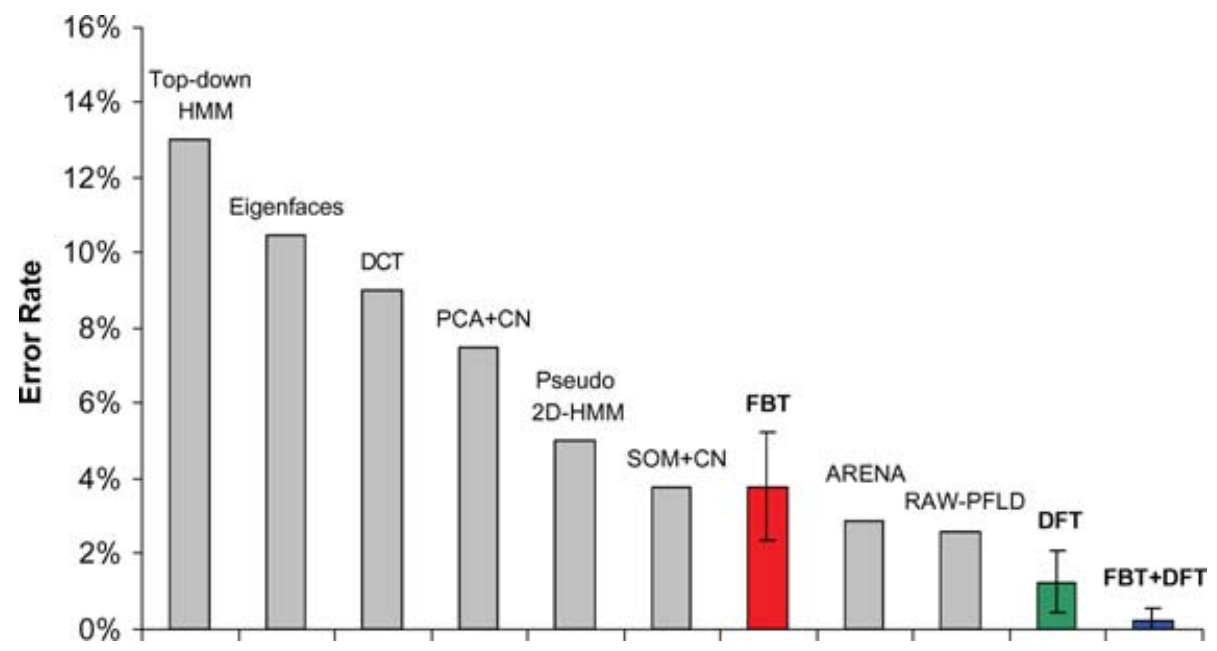

Fig. 7. Error rate of FBT, DFT, and FBT + DFT algorithms. Training size was five images per subject. Error bars indicate \pm 1 standard error of the mean. Results of previous algorithms are from Samaria and Harter [1994] (Top-down HMM); Samaria [1994] (Pseudo 2d-HMM); Lawrence et al. [1997] (Eigenfaces, PCA + CN, SOM + CN); Hafed and Levine [2001] (DCT); Sim et al. [2000] (ARENA); Pekalska and Duin [2000] (RAW-PFLD).

Harter 1994; Samaria 1994; Lawrence et al. 1997; Sim et al. 2000; Pekalska and Duin 2000; Hafed and Levine 2001]. With five images per subject, the FBT achieved a final performance of $3.8 \%$, while the DFT had an error rate of $1.25 \%$. However, when the FBT algorithm and the DFT where combined, the error rate was greatly reduced to $0.2 \%$. This means that, on average, of every 500 test images, only one was misclassified, which is an indication that the classification errors made by the FBT and DFT are successfully complementary. The performance of the FBT is equal to that achieved by the SOM-CN algorithm, but lower than that of the most successful methods (ARENA and RAW-PFLD). However, the DFT and the FBT + DFT algorithms outperformed all the others. 


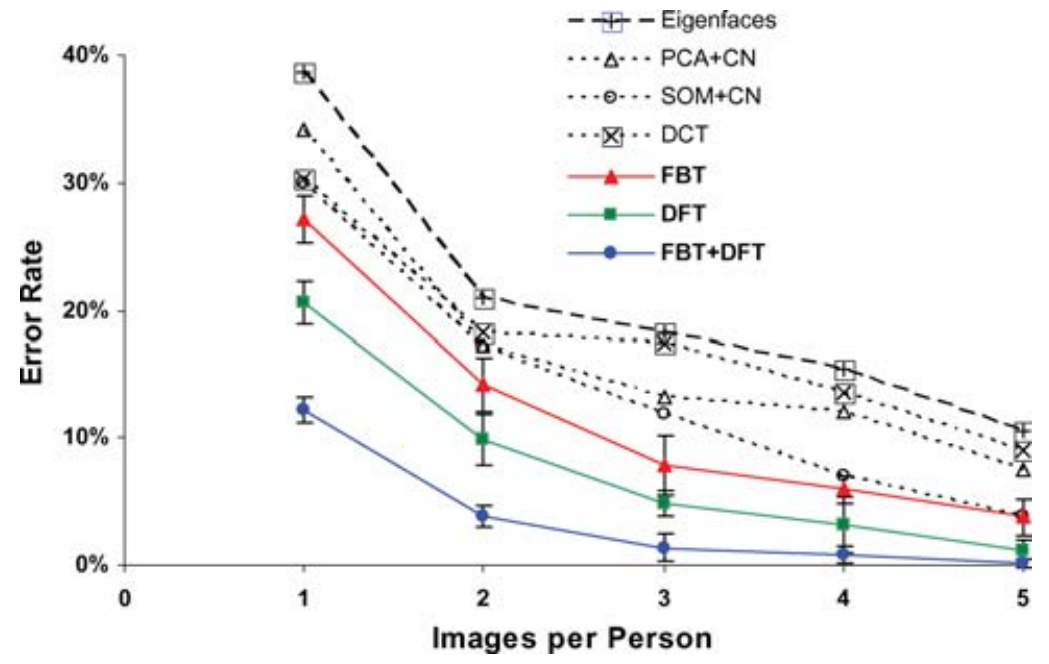

Fig. 8. Error rate as a function of the number of images learned from each subject. Error bars indicate \pm 1 standard error of the mean. . Results of previous algorithms are from Lawrence et al. [1997] (Eigenfaces, PCA + CN, SOM + CN); Hafed and Levine [2001] (DCT).

Figure 8 shows the error rate as a function of the number of images from each subject, i.e., the learning rate. For all cases, the error rate decreases as the number of training samples increase, as expected, since the increasing number of different samples from each subject increases the chances of correct recognition when presented with a new image. The performance of both FBT and DFT algorithms, for training sets of less than five images per subject, was superior to all previous algorithms tested in the same modality. However, the combined FBT + DFT classifier again achieved the lowest error rate.

Lai et al. [2001] developed a face-recognition system based on a spectroface representation, i.e., a wavelet transform and global Fourier invariant features, and presented results for the ORL database [Lai et al. 2001]. Their system achieved a 5.36\% error rate when the training set included three images per subject. This performance falls between those of the FBT and DFT. However, their results are optimistically biased, as the three training images were selected so as to form the best face representation.

5.1.3 Dataset Size Effect. The performance of face-recognition algorithms usually degrades as more subjects are added to the database, because of the increasing probability of the presence of subjects with similar attributes. This effect was confirmed with the models presented here (Figure 9). A comparable test on the same database was available only for the the SOM + CN algorithm [Lawrence et al. 1997]. Again, the performance of the FBT, DFT and the combined classifier were found to be superior.

5.1.4 Performance of Individual Features. We have also analyzed the performance of the FBT and DFT individual features. For this test, the number of extracted coefficients was extended. The training and test sets were composed of five images per subject, each image represented by a single feature. Recognition was based on a simple nearest-neighbor criterion, where a test image is assigned to the subject of the closest training image. Figure 10 shows the error rates obtained by using each feature. The highest performance for the FBT was obtained by coefficients in approximately the 0-20 Bessel order range coupled to Bessel roots in the 1-6 range. This result indicates that angular components of low-to-medium frequencies coupled to low-frequency radial components are the most informative with respect to the subject identity. The observation that the necessary features for good recognition 


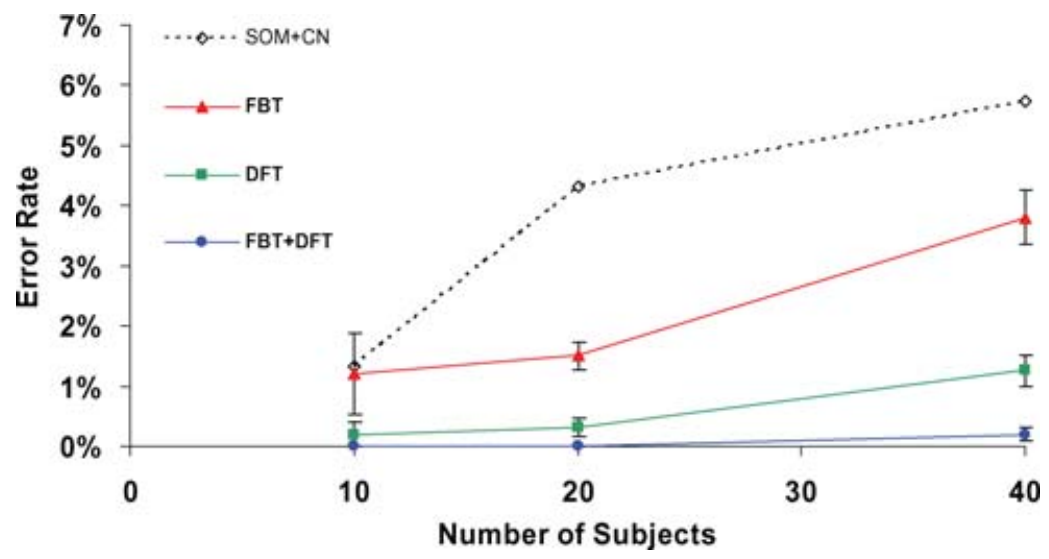

Fig. 9. Error rate as a function of the number of subjects in the database. Training set size was five images per person in all cases. Error bars indicate \pm 1 standard error of the mean. Results for the SOM + CN algorithm are from Lawrence et al. [1997].
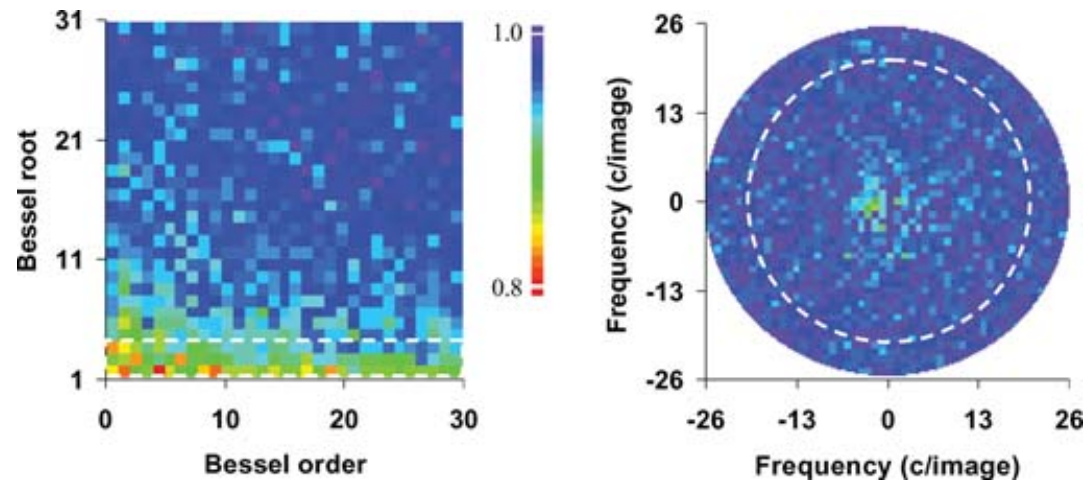

Fig. 10. Error rate, in colored levels, of individual features extracted by FBT (left panel) or (right panel) DFT. The DFT is presented in a conventional polar plot, where distance from the center represents frequency and angle represents orientation. Training size was five images per subject. Each pixel represents the average of 10 samples. The dashed white lines mark the spectral regions that were used in the FBT and DFT algorithms.

performance are insufficient to reconstruct the original image (Figure 4) agrees with previous studies [Turk and Pentland, 1991; Hafed and Levine, 2001] and does not violate any biological principle.

The DFT features presented a somewhat different behavior. The best performing features were distributed at all frequencies and orientations, although more concentrated in the low-frequency range and horizontal orientation ( $\mathrm{y}$ axis in the plot). This result may explain why the DFT algorithm requires such an extensive number of features, as compared to the FBT, to achieve good recognition levels.

\subsection{The FERET Database}

5.2.1 Database and Performance Evaluation. The FERET database was collected as part of the Face-Recognition Technology program to support algorithm development and evaluation. The main advantages of this database are the large number of individuals and rigid testing protocols that allow precise performance comparisons between different algorithms. Basically, a "gallery" set of one frontal view image from 1196 subjects is used to train the algorithm and a different dataset is used as probe. 

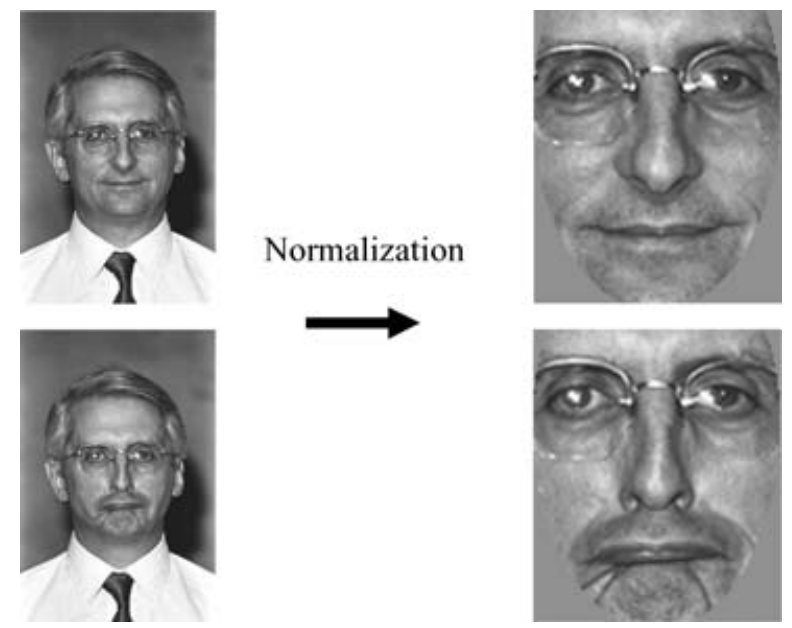

Fig. 11. Example of a normalization of two images taken from the same subject. The original images were scaled, aligned, centralized, cropped, and masked.

All images are gray-scale $256 \times 384$ pixels size. For the current study, we used the largest probe set, termed "FB," which is constituted of a single image from 1195 subjects. The probe set images were taken from the same subjects in the gallery set, after an interval of a few seconds, but with a different facial expression.

The original images were normalized using the eyes ground-truth information supplied with the database. Images were translated, rotated, and scaled so that the eyes were registered at specific pixels. Next, the images were cropped to $118 \times 140$ pixels size and a mask was applied to remove most of the hair and background (Figure 11). No histogram equalization or luminance normalization was performed, once these operations showed no performance improvement in our tests (Figure 11).

The performance of the algorithms was evaluated by identification and verification tests according to the FERET protocol [Phillips et al. 1998]. In the identification test, the algorithm ranks the gallery images according to their similarity to the probe images. A good algorithm would give a low ranking score to the correct matching images. For example, if the correct match of a probe image is among the five most similar gallery images, the matching is ranked five. Results are plotted as the proportion of correct identification as a function of the rank. Thus, the performance of an algorithm that matches 80 out of 100 probe images with ranking of five or less will be $80 / 10=0.8$ at rank 5 .

The verification test is based on an open-universe model. Given a gallery image $g$ and a probe image $p$, the algorithm verifies the claim that both were taken from the same subject, i.e., that $p=g$. The verification probability $P_{v}$ is the probability of the algorithm accepting the claim when it is true and the false-alarm rate $P_{F}$ is the probability of incorrectly accepting a false claim. The algorithm decision depends on a "confidence" ("posterior probability") score $s_{i}(k)$ given for each match and on a threshold $c$. Thus, a claim is confirmed if $s_{i}(k) \leq c$ and rejected otherwise. A plot of all the combinations of $P_{V}$ and $P_{F}$ as a function of $c$ is known as a receiver-operating characteristic (ROC). For each probe image, we computed the posterior probabilities for all the subjects in the gallery set and applied a threshold function. The results were split to a subset that consisted of all matches, where $p \approx g$ and a second subset with all matches where $p \neq g . P_{V}$ and $P_{F}$ were calculated as the number of confirmations divided by the number of matches in each subset, respectively. This procedure was repeated for 100 equally spaced threshold levels. 
5.2.2 Face Identification and Verification. Figure 12(A) shows the correct identification rate as a function of the rank for the proposed methods. In the same plot, we present results for the main previous algorithms, as published in Phillips et al. [1998]. The performance of the FBT algorithm was slightly superior to the basic PCA method, but lower than the state-of-the-art algorithms. In contrast to the results on the ORL database, here the DFT algorithm performed significantly worse than the FBT and no improvement was achieved by using the combined algorithm.

We also tested the proposed algorithms in a verification modality (Figure 12(B)). In this case, the FBT algorithm outperformed the PCA and the PCA + Bayesian algorithms. It equaled the Gabor + EBGM method at 0.005 false acceptance probability level, but did not reach the PCA + LDA performance. An interesting performance indicator is the equal error rate, i. e., the point where incorrect rejection and false alarm rates are equal. Figure 13 shows the results for the FBT algorithm in detail, while Table II summarizes results from previous studies. A comparative analysis of the equal error rate shows an even better performance of the FBT method: the error rate was lower than the PCA and PCA + Bayesian algorithms, equal to the Gabor + EBGM, but higher that of the PCA + LDA method. Here again, the DFT and the combined algorithm did not improve the performance over the FBT algorithm.

\section{DISCUSSION}

We introduced here a biologically motivated novel combination of techniques for face-recognition tasks. The new algorithm achieved considerable performance, although not surpassing that of state-of-the-art algorithms under all test conditions. We believe that it works because of the highly informative value of the polar frequency components, the conservation of proximity relations in the dissimilarity space, and by the efficient class separation provided by the linear discriminant.

\subsection{Polar Frequency Domain}

Although the relation of the present algorithm to human face recognition was not directly evaluated here, a few associations can be made. As discussed in the introduction, there is clear evidence that the HVS performs a Cartesian local analysis of the visual scene, but also pools this information to extract global radial and angular shapes. A model of such spatial processing was developed by Wilson and others [Wilson 1991; Wilson et al. 1997, 2001; Wilson and Wilkinson 1998, 2001]. Thus, in theory, the global polar frequency content of face images is available to the HVS, in addition to the local Cartesian frequency content.

In the current proposal, we explored an analogous global polar pooling by applying a FBT. The analogy resides not in the way the Cartesian information is pooled to extract global components, but in the coordinate definition of the fundamental global patterns. The face-recognition tests showed that only as little as 186 components are required for good performance, demonstrating the compactness of the polar representation. When tested on the ORL database, the FBT algorithm had achieved good performance compared to previous algorithms, but had the best performance when combined with a DFT-based algorithm. These results indicate coding of complementary information regarding face identity by the two transformation types. However, when tested on the larger FERET database, no performance gain was observed. These results suggest that the DFT contributed to the FBT performance by compensating for image variations such as translation, considering that the ORL images were not normalized, in contrast to the FERET images. This question should be addressed more specifically by future research.

The specific polar components can also be related to human performance. It was shown that the most informative individual features were found in the low-to-medium angular frequencies coupled to low radial frequencies, and that the FBT algorithm achieved good recognition performance when it was based on a wide range of angular frequencies coupled to low radial frequencies. We can not 

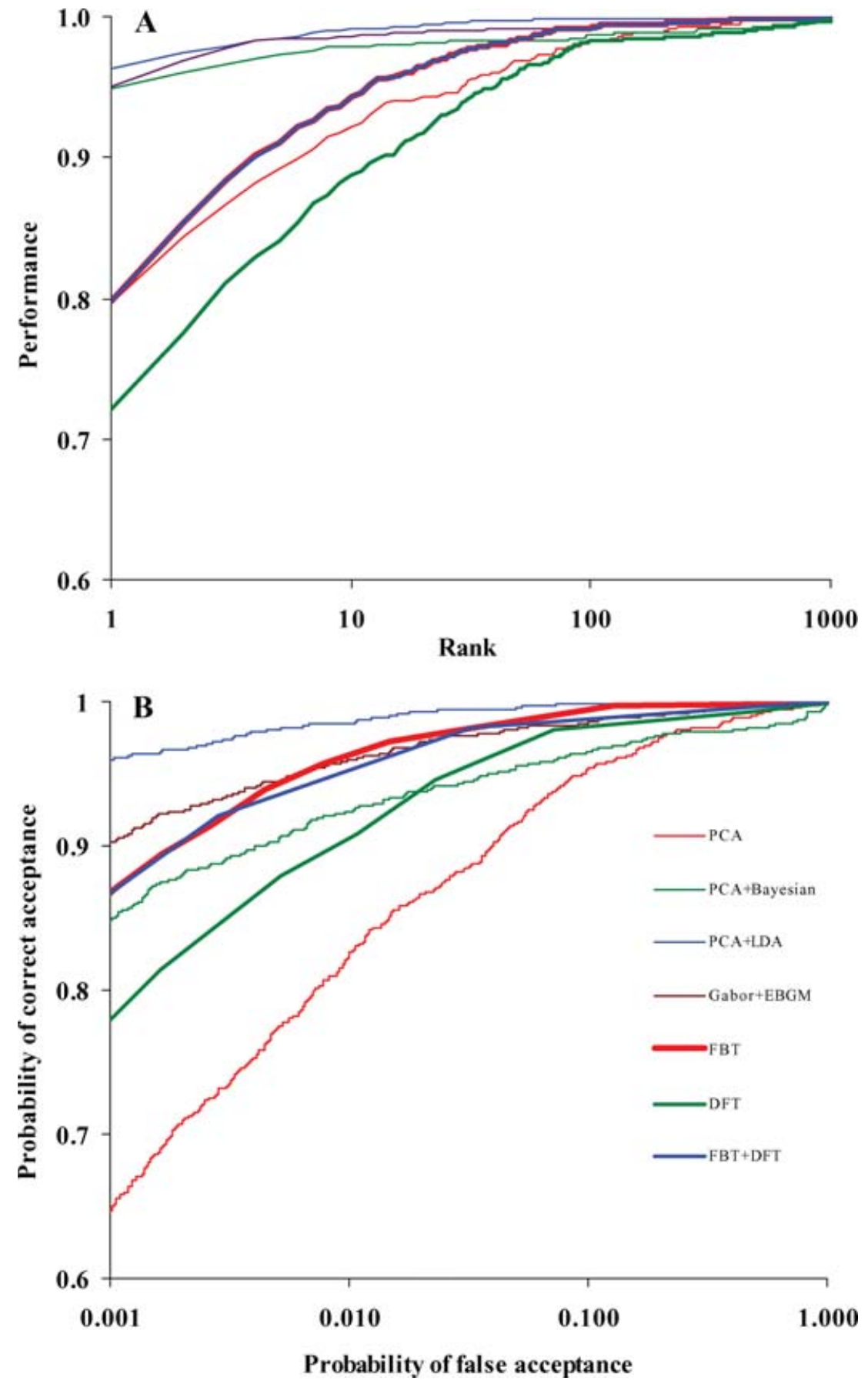

Fig. 12. Performance on a (A) ranked recognition and (B) verification tests. Results of previous algorithms are from Phillips et al. [1998], based on standard PCA (PCA) and works of Moghaddam et al. [2000] (PCA + Bayesian), Etemad and Chellappa [1997](PCA + LDA), and Lades et al. [1993](Gabor + EBGM). 


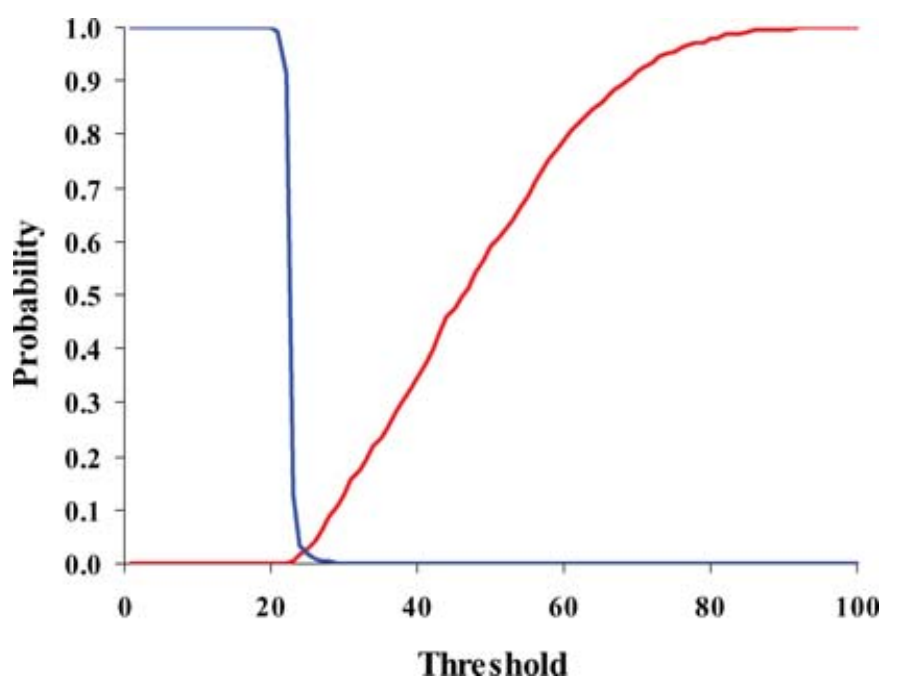

Fig. 13. Incorrect rejection (red line) and false alarm (blue line) rate as a function of the threshold of the FBT algorithm.

Table II. Equal Error Rate of the

Proposed and Previous Algorithms

\begin{tabular}{l|c}
\hline Algorithm & Equal Error Rate \\
\hline PCA & 7 \\
PCA + Bayesian & 4 \\
PCA + LDA & 1 \\
Gabor + EBGM & 2 \\
FBT & 2 \\
DFT & 4 \\
FBT + DGT & 3 \\
\hline
\end{tabular}

make a direct comparison of this finding with previous studies, since only Cartesian frequencies were considered in the latter case. It was evident, however, that the DFT-based algorithm required a wide range of frequency sampling in order to achieve good results, in agreement with the findings that both low and high spatial frequencies are important for face-recognition algorithms [Harmon, 1973; Sergent, 1986; Nastar, et al. 1997]. Human face recognition, on the other hand, is tuned to a limited band of frequencies, between 10 and 20 cycles per face, according to psychophysical studies [Tieger and Ganz 1979; Costen et al. 1996; Nasanen 1999]. Other studies concluded that gender classification and identification can be based on solely low- or high-frequency components, respectively [Sergent 1986]. Thus, if polar frequency components participate in human face recognition, it is expected that a wide range of angular frequencies, along with low radial frequencies, would have the highest relative weight for face recognition.

\subsection{Dissimilarity Representation Space}

In the proposed algorithm, the classifier operates in nondomain-specific metric space whose coordinates are similarity relations. In this space, images are represented as points whose coordinates are defined by their similarities to the other images, not by their FB coefficients. The effect of the PFLD is basically to reduce the distances between points of the same subjects and to increase it for points from other subjects. Probe images are also mapped onto this dissimilarity space, projected on the PFLD components, and classified according to the nearest class object. The high performance achieved by this representation 
indicates that the "real-world" proximity relations between face images are preserved to a good extent in the constructed internal space. An interesting question to ask is "Do humans internally represent faces in a dissimilarity space"?

One strategy to answer this question is to measure the dissimilarity (or distance) between different shape objects by objective (like geometry or pose) and perceptual (like recognition response time) parameters [for detailed review of shape representation by humans, see Edelman 1999]. Comparison of the two measurements is usually done by a multidimensional scaling (MDS) analysis, which projects objects as points in a two-dimensional space where the distance between the points approximate the Euclidean distance between the original objects. For example, in one study, 41 face images were sorted by degree of similarity according to geometrical (manual) measurements and by perceptual similarity [Rhodes 1988]. The two sorting method results were highly correlated, especially when the objective sorting used global features, such as age and weight of the persons in the images. Similar results were obtained in a neurophysiological study [Young and Yamane 1992] in which monkeys were presented with face images. It was found that the MDS proximity maps obtained from the original images and from the response patterns of neurons in the inferotemporal cortex had similar patterns.

Evidence of dissimilarity representation by humans comes also from studies that did not involve face images. In one of the first applications of this method, subjects were submitted to memory recall tests of the outline shapes of 15 of the US states [Shepard and Chipman 1970]. In other studies, subjects were submitted to recognition tests of closed contours representing the outline shape of objects [Shepard and Cermak 1973; Cortes and Dyre 1996], while objective measurements were based on Fourier descriptors. In all these cases, MDS analysis of shapes and subject responses revealed a similar pattern. Extending the studies to animal-like shapes led to the same conclusion [Edelman 1995; Cutzu and Edelman 1996, 1998]. The task of the subjects was to decide if two objects are similar and which of two pairs of objects are more similar to each other. Again, based on delay-time measurements, MDS analysis showed that people related the objects according to the objective similarity relations. Control experiments with "scrambled" (nonsense) shapes did not replicate the results.

These results indicate that representing images in a dissimilarity space, as done in the proposed algorithm, can be analogous to human representation mechanisms. It is important to note that in several of these studies, the shape measurements that correlated with the human performance were taken from a global perspective (age, Fourier descriptors, etc.) - a further indication of the relevance of global image analysis approach adopted here for human face recognition.

\subsection{Future Research}

Ideally, the representation of a face would be robust to changes in its appearance, but still be able to distinguish it from faces of other subjects. The FBT-based algorithm was tested only on frontal view face images and relatively constant illumination. There is no reason to believe that it will perform well under strong variation of this kind. From the computational point of view, we expect that a facetracking algorithm [Feris et al. 2004], followed by image normalization, would improve the algorithm performance and make it independent of ground-truth information. Preliminary tests also indicate that image resolution can be strongly reduced with only a small effect on performance, but with a significant reduction in processing time.

Performance improvement can be achieved by normalization of the FBT coefficients, such that the face representation becomes invariant to translation, rotation, and scale [Cabrera et al. 1992]. This is possible if the transform is obtained from the center of a face whose contour (or radius) is known. The coefficients ranking test results indicate that the algorithm may benefit from an implementation of a feature selection step. Currently all the coefficients in a specific range of frequencies are used. An alternative would be to increase the frequency range, but select only the coefficients with the highest 
predictive value, based on the training set. Finally, as the Euclidean distance currently in use was found to perform poorly [Sim et al. 2000; Moghaddam et al. 2000], other alternative metrics, like the Mahalanobis distance, should be evaluated.

An important issue to be investigated regards local and multiscale features, which should be integrated in our future experiments by applying local FBT and exploring Gabor filters and wavelets on polar representation of faces. Finally, our ongoing interests include face recognition in video sequences and in different $3 \mathrm{D}$ poses.

\section{ACKNOWLEDGMENTS}

This research was supported by grants from $\operatorname{CNPq}(150459 / 2003-3,301290 / 00-8,478384 / 2001-7,300722 /$ 98-2) and FAPESP (No: 03/07519-0 and 99/12765-2). We are grateful to Paul Fox, Viviana Giampaoli, Bob Duin, Elzbieta Pekalska, and Jesus Mena-Chalco for their valuable comments on the mathematical aspects of the work. We are also grateful to the reviewers for the useful comments that helped us to improve the paper.

\section{REFERENCES}

Allwein, E. L., Schapire, R. E., AND Singer, Y. 2000. Reducing multiclass to binary: a unifing approach for margin classifiers. Journal of Machine Learning Research 1, 113-141.

Arca, A., Campadelli, P., ANd Lanzarotti, R. 2003. A face recognition system based on local feature analysis. Lecture Notes in Computer Science 2688, 182-189.

AвDI, H. 1988. A generalized approach for connectionist auto-associative memories: interpretation, implication and illustration for race process. In Artificial Intelligence and Cognitive Sciences, J. Demongeot, T. Hervé, V. Rialle, and C. Roche, Eds. Manchester University Press, Manchester. 149-165.

Akamatsu, H. F. S., Sasaki, T., And Suenuga, Y. 1991. A robust face identification scheme--KL expansion of an invariant feature space. In SPIE Proceedings: Intelligent Robots and Computer Vision X: Algorithms and Techniques 1607. 71-84.

Bowman, F. 1958. Introduction to Bessel Functions. Dover, New York.

Cabrera, J., Falcón, A., Hernández, F. M., and MÊndez, J. 1992. A systematic method for exploring contour segment descriptions. Cybernetics and Systems 23, 241-270.

Calder, A. J., Burton, A. M., Miller, P., Young, A. W., And Akamatsu, S. 2001. A principal component analysis of facial expressions. Vision Research 41, 1179-1208.

Campbell, F. W. and Robson, J. G. 1968. Application of Fourier analysis to the visibility of gratings. Journal of Physiology 197, $551-566$.

Chien, S. I. And Choi, I. 2000. Face and facial landmarks location based on log-polar mapping. Lecture Notes in Computer Science 1811, 379-386.

Cortes, J. M. And Dyre, B. P. 1996. Perceptual similarity of shapes generated from Fourier Descriptors. Journal of Experimental Psychology: Human Perception and Performance 22, 133-143.

Costen, N. P., Parker, D. M., and Craw, I. 1996. Effects of high-pass spatial filtering on face identification. Perception and Psychophysics 58, 602-612.

Craw, I. and Cameron, P. 1991. Parametising images for recognition and reconstruction. Proceedings of the British Machine Vision Conference. 367-370.

Cutzu, F. and Edelman, S. 1996. Faithful representation of similarities among three-dimensional shapes in human vision. Proceedings of the National Academy of Science 93, 12046-12050.

Cutzu, F. and Edelman, S. 1998. Representation of object similarity in human vision: psychophysics and a computational model. Vision Research 38, 2229-2257.

De Valois, R. L. and De Valois, K. K. 1990. Spatial Vision. Oxford University Press, New York.

De Valois, R. L., Yund, E. W., ANd Helper, N. 1982. The orientation and direction selectivity of cells in macaque visual cortex. Vision Research 22, 531-544.

DuIn, R. P. W. 2000. PRTools_3, A Matlab Toolbox for Pattern Recognition. Delft University of Technology, Delft (Holland).

Duin, R. P. W., De Ridder, D., ANd TAX, D. M. J. 1997. Experiments with a featureless approach to pattern recognition. Pattern Recognition Letters 18, 1159-1166.

Edelman, S. 1995. Representation of similarity in 3D object discrimination. Neural Computation 7, 407-422. 
Edelman, S. 1999. Representation and Recognition in Vision. MIT Press, Cambridge.

Escobar, M. J. ANd Ruiz-DEL-solar, J. 2002. Biologically-based face recognition using Gabor filters and log-polar images. Proceedings of the International Joint Conference on Neural Networks 2, 1143-1147.

Etemad K. and Chellappa R. 1997. Discriminant analysis for recognition of human face images. Journal of the Optical Society of America A-Optics Image Science and Vision 14, 1724-1733.

Feris, R. S., Krueger, V., ANd CeSAR-JR., R. M. 2004. A wavelet subspace method for real-time face tracking. Journal of RealTime Imaging 10, 339-350.

Fox, P. D. 2000. Computation of linear ultrasound fields using 2D Fourier-Bessel series. Proceedings of the 25th International Acoustical Imaging Symposium. 19-22.

Fox, P. D., Cheng, J., AND Lu, J. 2003. Theory and experiment of Fourier-Bessel field calculation and tuning of a pulsed wave annular array. Journal of the Acoustical Society of America 113, 2412-2423.

Fukunaga, K. 1990. Introduction to Statistical Pattern Recognition. Academic Press, New York.

Gallant, J. L., Braun, J., And Vanessen, D. C. 1993. Selectivity for polar, hyperbolic, and Cartesian gratings in macaque visual cortex. Science 259, 100-103.

Gallant, J. L., Connor, C. E., Rakshit, S., Lewis, J. W., and Vanessen, D. C. $1996 . \quad$ Neural responses to polar, hyperbolic, and Cartesian grating in area V4 of the macaque monkey. Journal of Neurophysiology 76, 2718-2739.

Gomes, H. M. ANd Fisher, R. B. 2003. Primal sketch feature extraction from a log-polar image. Pattern Recognition Letters 24, 983-992.

Grove, T. D. And Fisher, R. B. 1996. Attention in iconic object matching. Proceedings of the British Machine Vision Conference 1, Edinburgh. 293-302.

Guan, S., LaI, C. H., ANd We, G. W. 2001. Fourier-Bessel analysis of patterns in a circular domain. Physica D 151, 83-98.

Hafed, Z. M. ANd Levine, M. D. 2001. Face recognition using the discrete cosine transform. International Journal of Computer Vision 43, 167-188.

Harmon, L. D. 1973. The recognition of faces. Scientific American 229, 71-82.

Hotta, K., KuRita, T., AND Mishima, T. 1998. Scale invariant face detection method using higher-order local autocorrelation features extracted from log-polar image. Proceedings of the 3rd IEEE International Conference on Automatic Face and Gesture Recognition, 70-75.

Hubel, D. H. ANd Wiesel, T. N. 1968. Receptive field and functional architecture of monkey striate cortex. Journal of Physiology $195,215-243$.

Itтi, L., Kосн, C., AND Braun, J. 2000. Revisiting spatial vision: Toward a unifying model. Journal of the Optical Society of America A17, 1899-1917.

JURIE, F. 1999. A new log-polar mapping for space variant imaging: Application to face detection and tracking. Pattern Recognition 32, 865-875.

Kelly, D. H. 1960. Stimulus pattern for visual research. Journal of the Optical Society of America 50, 1115-1116.

Kittler, J., Hatef, M., Duin, P. W., and Matas, J. 1998. On combining classifiers. IEEE Transactions on Pattern Analysis and Machine Intelligence 20, 226-239.

Lades, M., Vorbruggen, J. C., Buhmann, J., Lange, J., Vandermalsburg, C., Wurtz, R. P., And Konen, W. 1993. Distortion invariant object recognition in the dynamic link architecture. IEEE Transactions on Computers 42, 300-311.

Lai, H. L., Yuen, P. C., And Feng, G. C. 2001. Face recognition using holistic Fourier invariant features. Pattern Recognition $34,95-109$.

Lawrence, S., Giles, C. L., Tsoi, A. C., ANd Back, A. D. 1997. Face recognition: A convolutional neural network approach. IEEE Transactions on Neural Networks 8, 98-113.

Lim, F. L., West, G. A. W., and Venkatesh, S. 1997. Use of log-polar space for foveation and feature recognition. IEEE Proceedings-Vision Image and Signal Processing 144, 323-331.

Mahon, L. E. and De VAlois, R. L. 2001. Cartesian and non-Cartesian responses in LGN, V1, and V2 cells. Visual Neuroscience $18,973-981$.

Minut, S., Mahadevan, S., Henderson, J., And Dyer, F. 2000. Face recognition using foveal vision. Lecture Notes in Computer Science-LNCS 1811. 424-433.

Moghaddam, B., Jebara, T., and Pentland, A. 2000. Bayesian face recognition. Pattern Recognition 33, 1771-1782.

Movshon, J. A., Thompson, I. I., And Tolhurst, D. J. 1978. Spatial and temporal contrast sensitivity of neurons in areas 17 and 18 of the cat's visual cortex. Journal of Physiology (London) 283. 79-99.

NASANEN, R. 1999. Spatial frequency bandwidth used in the face recognition of facial images. Vision Research 39, $3824-3833$. 
Nastar, C., Moghaddam, B., and Pentland, A. 1997. Flexible images: Matching and recognition using learned deformations. Computer Vision and Image Understanding 65, 179-191.

Obermayer, K., Blasdel, G. G., AND Schulten, K. 1991. A neural network model for the formation and for the spatial structure of retinotopic maps, orientation and ocular dominance columns. In Artificial Neural Networks, T. Kohonen, K. Mäkisara, O. Simula, and J. Kangas, Eds., Elsevier, Amsterdam, Netherlands, 505-511.

O'toole, A. J., Abdi, H., Deffenbacher, K. A., and Valentin, D. $1995 . \quad$ A perceptual learning theory of the information in face. In Cognitive and Computational Aspects of Face Recognition, T. Valentine, Ed. Routledge, London. 159-182.

Pekalska, E. And Duin, R. P. W. 2000. Classification on dissimilarity data: A first look. Proceedings of the 6th Annual Conference of the Advanced School for Computing and Imaging. 221-228.

PerRet, D. I., Rolls, E. T., AND CAAN, W. 1982. Visual neurons responsive to faces in the monkey temporal cortex. Experimental Brain Research 47, 329-342.

Phillips, P. J., Wechsler, H., Huang, J., and Rauss, P. 1998. The FERET database and evaluation procedure for face recognition algorithms. Image and Vision Computing Journal 16, 295-306.

Pratt, W. K. 1991. Digital Image Processing. Wiley-Interscience Pub., New York.

Raudy, S. J. ANd Pikelis, V. 1980. On dimensionality, sample size, classification error, and complexity of classification algorithms in pattern recognition. IEEE Transactions on Pattern Analysis and Machine Intelligence 2, 243-251.

RHODES, G. 1988. Looking at faces: First-order and second-order features as determinants of facial appearance. Perception 17, $43-63$.

Rosental, S., Davis, S. H., And Homsy, G. M. 1982. Nonlinear Marangoni convection in bounded layers. Part 1. Circular cylindrical containers. Journal of Fluid Mechanics 120, 91-122.

SAmaria, F. S. 1994. Face recognition using Hidden Markov Models. PhD thesis, Trinity College, University of Cambridge, Cambridge.

SAmaria, F. S. ANd Harter, A. C. 1994. Parameterization of a stochastic model for human face identification. Proceedings of the 2nd IEEE Workshop on Applications of Computer Vision. 138-142.

Schwartz, E. L. 1977. Spatial mapping in primate sensory projection: Analytic structure and relevance to perception. Biological Cybernetics 25, 181-194.

Schwartz, E. L. 1980. Computational anatomy and functional architecture of striate cortex: A spatial mapping approach to perceptual coding. Vision Research 20, 645-669.

Scurichina, M. AND Duin, R. P. W. 1996. Stabilizing classifiers for very small sample sizes. Proceedings of the 13th International Conference on Pattern Recognition 2, Track B. 891-896.

Sergent, J. 1986. Microgenesis of face perception. In Aspects of Face Processing., H. D. Ellis, M. A., Jeeves, F. Newcombe, and A. Young, Eds. Nijhoff, Dordrecht, The Netherlands.

Shepard, R. N. And Cermak, G. W. 1973. Perceptual-cognitive explorations of a toroidal set of free-form stimuli. Cognitive Psychology 4, 351-377.

Shepard, R. N. And Chipman, S. 1970. Second-order isomorphism of internal representations: Shapes of states. Cognitive Psychology 1, 1-17.

Sim, T., Sukthankar, R., Mullin, M., and Baluja, S. 2000. Memory-based recognition for visitor identification. Proceedings of the 4th International Conference on Automatic Face and Gesture Recognition. 214-220.

Smeraldi, F. And Bigun, J. 2002. Retinal vision applied to facial features detection and face authentication. Pattern Recognition Letters 23, 463-475.

Spanier, J. ANd Oldham, K. B. 1987. An Atlas of Functions. Hemisphere Pub. Corp., Washington, DC.

Tieger, T. And Ganz, L. 1979. Recognition of faces in the presence of two-dimensional sinusoidal masks. Perception and Psychophysics $26,163-167$.

Tistarelli, M. and Grosso, E. 1998. Active vision-based face recognition issues, applications and techniques. In Nato-Asi Advanced Study on Face Recogniton, Wechsler, H., et al. Eds., Vol. F-163. Springer, Berlin. 262-286.

Turk, M. and Pentland, A. 1991. Eigenfaces for recognition. Journal of Cognitive Neuroscience 3, 71-86.

Wilson, H. R. 1991. Psychophysical models of spatial vision and hyperacuity. In Spatial Vision D. Regan, Ed., Macmillan: London. 64-86.

Wilson, H. R. AND Wilkinson, F. 1998. Detection of global structure in Glass patterns: Implications for form vision. Vision Research 38, 2933-2947.

Wilson, H. R., Wilkinson, F., ANd AsaAd, W. 1997. Concentric orientation summation in human form vision. Vision Research $37,2325-2330$. 
Wilson, H. R., Loffler, G., Wilkinson, F., and Thistlethwaite, W. A. 2001. An inverse oblique effect in human vision. Vision Research 41, 1749-1753.

Wiskott, L., Fellous, J., Krüger, N., And Von Der Malsbnurg, C. 1997. Face recognition by elastic bunch graph matching. IEEE Transactions on Pattern analysis and Machine Intelligence 19, 775-779.

Young, M. P. And Yamane, S. 1992. Sparse population coding of faces in the inferotemporal cortex. Science 256, 1327-1331.

Zwick, M. AND Zeitler, E. 1973. Image reconstruction from projections. Optik 38, 550-565.

Received February 2004; revised August 2004; accepted November 2005 